\title{
Temporal Dynamics of Brain White Matter Plasticity in Sighted Subjects during Tactile Braille Learning: A Longitudinal Diffusion Tensor Imaging Study
}

\author{
Malwina Molendowska, ${ }^{1,2 *}$ Jacek Matuszewski, ${ }^{1 *}$ Bartosz Kossowski, ${ }^{1}$ Lukasz Bola, ${ }^{1,3}$ Anna Banaszkiewicz, ${ }^{1}$ \\ Małgorzata Paplińska, ${ }^{4}$ Katarzyna Jednoróg, ${ }^{5}$ Bogdan Draganski, ${ }^{6,7}$ and Artur Marchewka ${ }^{1}$ \\ ${ }^{1}$ Laboratory of Brain Imaging, Nencki Institute of Experimental Biology, Polish Academy of Sciences, 02-093, Warsaw, Poland, ${ }^{2}$ Cardiff University \\ Brain Research Imaging Centre (CUBRIC), Cardiff University, Cardiff CF24 4HQ, United Kingdom, ${ }^{3}$ Institute of Psychology, Jagiellonian University, \\ 30-060, Krakow, Poland, ${ }^{4}$ The Maria Grzegorzewska University, 02-353, Warsaw, Poland, ${ }^{5}$ Laboratory of Language Neurobiology, Nencki Institute \\ of Experimental Biology, Polish Academy of Sciences, 02-093, Warsaw, Poland, ${ }^{6}$ Laboratory for Research in Neuroimaging, Department of Clinical \\ Neurosciences, Lausanne University Hospital and University of Lausanne, CH-1015 Lausanne, Switzerland, and ${ }^{7}$ Neurology Department, Max \\ Planck Institute for Human Cognitive and Brain Sciences, D-04303 Leipzig, Germany
}

The white matter (WM) architecture of the human brain changes in response to training, though fine-grained temporal characteristics of training-induced white matter plasticity remain unexplored. We investigated white matter microstructural changes using diffusion tensor imaging at five different time points in 26 sighted female adults during 8 months of training on tactile braille reading. Our results show that training-induced white matter plasticity occurs both within and beyond the trained sensory modality, as reflected by fractional anisotropy (FA) increases in somatosensory and visual cortex, respectively. The observed changes followed distinct time courses, with gradual linear FA increase along the training in the somatosensory cortex and sudden visual cortex cross-modal plasticity occurring after braille input became linguistically meaningful. WM changes observed in these areas returned to baseline after the cessation of learning in line with the supply-demand model of plasticity. These results also indicate that the temporal dynamics of microstructural plasticity in different cortical regions might be modulated by the nature of computational demands. We provide additional evidence that observed FA training-induced changes are behaviorally relevant to tactile reading. Together, these results demonstrate that WM plasticity is a highly dynamic process modulated by the introduction of novel experiences.

Key words: DTI; longitudinal design; neuroplasticity; tactile reading; white matter

Significance Statement

Throughout the lifetime the human brain is shaped by various experiences. Training-induced reorganization in white matter (WM) microstructure has been reported, but we know little about its temporal dynamics. To fill this gap, we scanned sighted subjects five times during tactile braille reading training. We observed different dynamics of WM plasticity in the somatosensory and visual cortices implicated in braille reading. The former showed a continuous increase in WM tissue anisotropy along with tactile training, while microstructural changes in the latter were observed only after the participants learned to read braille words. Our results confirm the supply-demand model of brain plasticity and provide evidence that WM reorganization depends on distinct computational demands and functional roles of regions involved in the trained skill.

Received Aug. 26, 2020; revised May 5, 2021; accepted June 8, 2021.

Author contributions: K.J., B.D., and A.M. designed research; J.M., A.B., and M.P. performed research; M. M., B.K., and M.P. contributed unpublished reagents/analytic tools; M.M., J.M., B.K., L.B., K.J., and A.M. analyzed data; M.M., J.M., L.B., K.J., B.D., and A.M. wrote the paper.

This study was supported by National Science Center Poland Grants 2018/30/E/HS6/00206 and 2014/14/M/ HS6/00918 awarded to A.M. J.M. was additionally supported by National Science Center Poland Grant Preludium 2017/27/N/HS6/02669. The study was conducted with the aid of CePT research infrastructure purchased with funds from the European Regional Development Fund as part of the Innovative Economy Operational Program, 2007-2013.

${ }^{*}$ M.M. and J.M. contributed equally to this work.

The authors declare no competing financial interests.

Correspondence should be addressed to Artur Marchewka at a.marchewka@nencki.edu.pl.

https://doi.org/10.1523/JNEUROSCI.2242-20.2021

Copyright $\odot 2021$ the authors

\section{Introduction}

The human brain shows a substantial propensity for training/ learning-induced plastic changes beyond the developmental critical period (May and Draganski, 2008; Lövdén et al., 2010; Zatorre et al., 2012). Recent advances in noninvasive assessment of brain white matter (WM) suggest dynamic changes in response to training and learning (Lerch et al., 2017; Sampaio-Baptista and Johansen-Berg, 2017). A shortcoming of previous studies is the sparsity of longitudinal data acquisition, with many months between time points, which limits inferences on fine-grained temporal characteristics of training-induced white matter plasticity. 
A

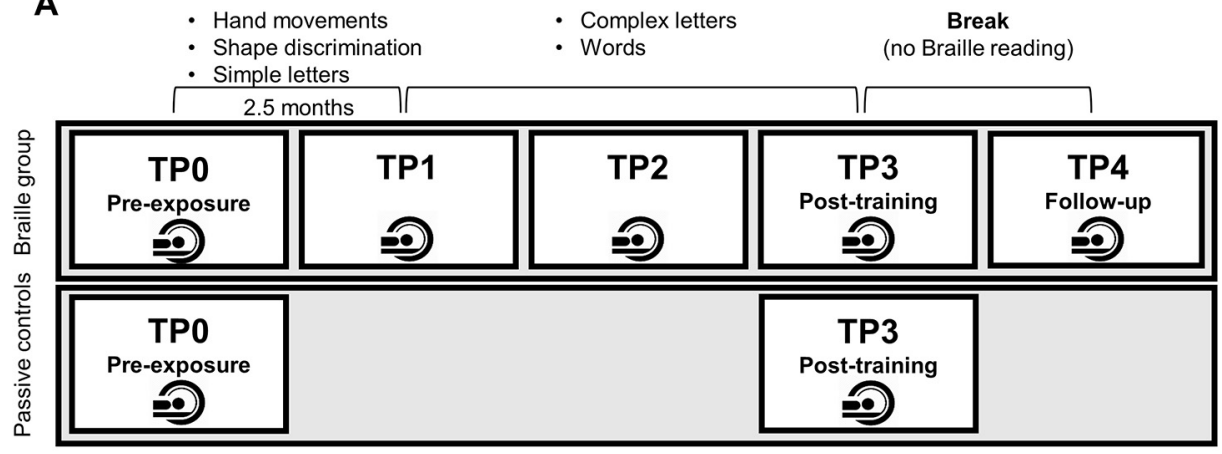

B

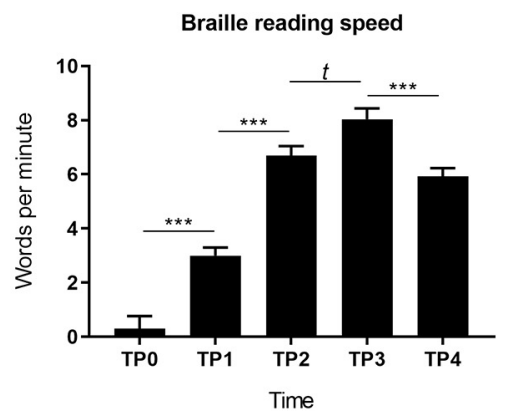

Figure 1. Experimental design and behavioral results. $\boldsymbol{A}$, Twenty-six subjects from the experimental group trained in tactile braille reading for 8 months and participated in five MRI and behavioral testing sessions performed 2.5 months apart. Twenty-five passive control subjects participated in baseline and post-training sessions. $\boldsymbol{B}$, Tactile braille reading speed over the course of training. Error bars represent SEMs adjusted for within-subject designs according to Cousineau (2005): $t=$ trend $\left(p_{\text {corr }}=0.066\right)$, ${ }^{* * *} p<0.001$, Bonferroni corrected.

In the WM, local intracortical connectivity constrains the type and the nature of neuronal computations, while long-range white-matter connections distribute information across different brain systems (Jbabdi et al., 2015). The study of microstructure, which in magnetic resonance imaging (MRI) refers to the description of the tissue microscale characteristic content at the voxel level, has been primarily the domain of diffusion MRI (Lerch et al., 2017). Diffusion tensor imaging (DTI) is the most common diffusion signal representation used to derive voxelbased scalar measures including fractional anisotropy (FA), axial diffusivity $(\mathrm{AD})$, radial diffusivity $(\mathrm{RD})$, or mean diffusivity (MD; Basser et al., 1994; Pierpaoli et al., 1996), of which FA is generally acknowledged in evaluation of microstructure-related changes (Snook et al., 2005; Lebel et al., 2019).

Neuroplasticity-the capacity of the brain to change in response to new environmental demands (Lövdén et al., 2010) has been studied in WM on various temporal scales ranging from hours to weeks of training. Sagi et al. (2012) showed that 2 $\mathrm{h}$ of video game training induces MD decreases in parahippocampal WM. Several weeks of training juggling (Scholz et al., 2009), body balancing (Taubert et al., 2010), unicycling (Weber et al., 2019), or verbal and spatial span tasks (Caeyenberghs et al., 2016; Metzler-Baddeley et al., 2017) lead to changes in FA. However, a large body of evidence comes from paradigms incorporating repetitive training schemes, usually related to motor domain and often including only two measurements (pretraining and post-training; Zatorre et al., 2012; Lövdén et al., 2013).

Braille reading is a cross-modal, sensory-motor skill involving language processing via tactile modality. Tactile reading involves evaluation of the input from finger sensation in the somatosensory cortex followed by basic spatial analysis of braille character dots in the early visual cortex and, finally, word form recognition in higher-order visual cortices (Zangaladze et al., 1999; Merabet et al., 2008; Bola et al., 2019). Previous studies have shown that teaching tactile braille to sighted adults results in functional and anatomic reorganization of the brain. Specifically, results of the study by Debowska et al. (2016) indicated that 3 weeks of training increased FA in the somatosensory system (including primary and secondary somatosensory cortex as well as thalamus) and visual cortex (lingual and fusiform gyri). In addition, Bola et al. (2017) showed that 9 months of tactile reading training led to $\mathrm{FA}$ increases paralleled by $\mathrm{RD}$ decreases in the early visual cortex. These studies included only pretraining and post-training scans; therefore, the dynamics of microstructural changes during learning of this complex skill remain unexplored.
Here, we address the aforementioned limitations by tracing WM changes on a more densely sampled time interval than the commonly applied two time points (pretraining/post-training) designs. We investigated experience-induced brain plasticity in 26 sighted female subjects participating in a tactile braille reading course of 8 months duration. We acquired behavioral and neuroimaging data during the learning phase across five time points. With this design, we conducted the following three types of analysis: (1) braille learning-specific structural reorganization compared with passive control group; (2) relations between WM structural properties and the proficiency in tactile reading over time; and (3) exploratory time course analysis. We hypothesized that the constant novel input during training should be reflected in FA increases in the somatosensory WM, followed by the microstructural reorganization of the visual cortex.

\section{Materials and Methods}

Experimental design. Over the course of tactile reading training, participants underwent five behavioral testing and brain imaging sessions $\sim 2.5$ months apart, as follows: pre-exposure [time point 0 (TP0)], two during learning (TP1, TP2), one right after finishing the training (TP3), and a follow-up study 2.5 months after the end of the training (TP4; Fig. $1 A)$. In addition, we included a passive control group that took part in TP0 and TP3 brain-imaging sessions. Subjects received financial reimbursement both for participation in a braille course and for each of the MRI sessions. Apart from T1-weighted and diffusion-weighted imaging, we analyzed fMRI correlates of neural activity related to tactile reading and quantitative $\mathrm{T} 1$ mapping data described in the study by Matuszewski et al. (2021).

Subjects. Thirty-two sighted, right-handed females (mean age, 23.2 years; SD, 3.3; age range, 19-32 years) took part in a tactile braille reading course and MRI sessions. Six of the participants were excluded because of either anatomic abnormalities detected in the first MRI session (two individuals) or dropping out of the study (four subjects). Thus, 26 individuals (mean age, 23.1 years; SD, 3.4; age range, 19-32 years) were included in the analyses. All individuals were native Polish speakers and had normal or corrected-to-normal vision and were naive to the tactile braille alphabet. Twenty-two of the participants were students or postgraduates of studies in special education of the blind, 3 were workers in a charitable foundation supporting blind people, and 1 was a parttime braille visual transcriber. Participants were familiar with the basics of visual braille. Informed consent forms and MRI safety screening results were collected before the study. The study was approved by the local Committee for Research Ethics. The present study participants are independent from those previously described by our group (Bola et al., 2016, 2017; Siuda-Krzywicka et al., 2016). However, 21 of them participated in our recently published study using transcranial magnetic stimulation (TMS; Bola et al., 2019). 
Additionally, 28 sighted, right-handed, female students (mean age, 23.3 years; SD, 2.1; age range, 19-27 years) were recruited as control subjects. Two subjects were excluded because of MRI artifacts assessed based on quality checks implemented in CAT12 SPM toolbox, and one subject was excluded because of within-subject average image creation failure, resulting in 25 subjects (mean age, 23.1 years; SD, 2.1; age range, 19-27 years) included in the analyses. All were native Polish speakers, had normal or corrected-to-normal vision and were naive to the braille alphabet. During the experiment, they did not participate in any training related to the acquisition of new language skills. Control subjects were demographically matched to the experimental group, with no statistically significant differences in age, education, or proficiency in foreign languages detected ( $p$ values $>0.05$ ).

Tactile reading course and performance tests. The tactile reading course lasted 8 months and involved meetings with an instructor and self-paced work with sets of 30 exercise cards per month. Meetings with the instructor were organized for the whole group two times per month in the first 2 months and in smaller groups (5-10 subjects) once per month subsequently. The self-paced learning was based on 30 cards given monthly, with each card representing $\sim 15-20 \mathrm{~min}$ of tactile reading. Subjects were instructed to read as regularly as possible. Because of the demanding nature of tactile reading, the course only covered 16 Polish letters (A, B, C, D, E, I, K, L, Ł, M, O, P, S, T, U, and Y). The first $10 \mathrm{~h}$ of the course involved basic principles of hand movements during braille reading as well as basic discrimination of shapes. Subsequently, letters and words were introduced, starting with easier letters $(A, B, C$, $\mathrm{D}, \mathrm{E}, \mathrm{I}, \mathrm{K}$, and $\mathrm{O}$ ), while the more difficult ones (L, $\mathrm{E}, \mathrm{M}, \mathrm{S}, \mathrm{P}, \mathrm{T}, \mathrm{U}$, and Y) were taught after 3 months of training (Fig. 1), followed by the reading of simple and complex words. For details on the tactile braille course for sighted subjects, see Bola et al. (2016). During the first 3 months of tactile training, 13 subjects reported their preference for reading with the left (L) hand despite being right (R) handed. This phenomenon was reported previously both in blind and sighted subjects (Sadato et al., 2002; Siuda-Krzywicka et al., 2016). However, since tactile reading involves using both hands simultaneously (with one hand supporting navigation between words and lines and the other one reading), actual tactile input is often similar but not identical (e.g., more fine-grained parsing of a sign is performed by the reading hand).

The tactile reading test consisted of 20 braille words (three to five letters long) with SUBTLEX-PL frequency occurrence over 1 per million (Mandera et al., 2015). The participants' task was to read aloud with their preferred hand as many words as they could within $60 \mathrm{~s}$ while being blindfolded. Only words read correctly were counted. Twelve different versions of tests were used, counterbalanced across subjects and matched for word length, and letters were introduced during the course.

MRI data acquisition. All MRI data were acquired using a $3 \mathrm{~T}$ Siemens Trio scanner with a 12-channel array head coil and a $40 \mathrm{mT} / \mathrm{m}$ diffusion gradient. For the T1-weighted anatomic scans, we used an MPRAGE sequence covering the whole brain. The protocol parameters were as follows: $\mathrm{TR}=2530 \mathrm{~ms} ; \mathrm{TI}=1100 \mathrm{~ms} ; \mathrm{TE}=3.32 \mathrm{~ms} ; \mathrm{FOV}=256$ $\times 256 \mathrm{~mm}$; flip angle $=7^{\circ}$; isotropic voxel $=1 \mathrm{~mm}^{3}$. Diffusion-weighted images (DWIs) were collected using diffusion-weighted spin echo EPI pulse sequence $(\mathrm{TR}=12,500 \mathrm{~ms} ; \mathrm{TE}=93 \mathrm{~ms} ; \mathrm{FOV}=220 \times 220 \mathrm{~mm}$; slice thickness $=2 \mathrm{~mm}$; matrix size $=128 \times 128$; in-plane resolution $=1.72 \times 1.72$ $\mathrm{mm}^{2}$; slices $=80 ; b=1500 \mathrm{~s} / \mathrm{mm}^{2}$; diffusion gradient directions $=64$; generalized autocalibrating partial parallel acquisition factor $=2$ ), and two nondiffusion-weighted images $\left(b=0 \mathrm{~s} / \mathrm{mm}^{2}\right)$ with AP (anterior - posterior) phase encoding direction were also collected. Immediately after obtaining diffusion-weighted images, field maps were acquired to correct the data for magnetic field inhomogeneities artifacts $(\mathrm{TR}=700 \mathrm{~ms}$; TE1 $=4.39 \mathrm{~ms}$; $\mathrm{TE} 2=6.85 \mathrm{~ms}$; flip angle $=60^{\circ}$; slices $=68$; slice thickness $=2 \mathrm{~mm}$; FOV and in-plane resolution are the same as for the DWI).

Data processing. For voxel-based statistical analyses, MRI data were processed using MRTrix complemented with FSL, Advanced Normalization Tools [ANTs (http://stnava.github.io/ANTs/); Avants et al., 2011; Andersson and Sotiropoulos, 2016] and SPM12 [Statistical Parametric Mapping (http://www.fil.ion.ucl.ac.uk/spm/)] toolbox. We first calculated white matter tissue probability maps across all study participants and across all time points. For each subject, an average ("within-subject") bias-corrected T1-weighted image was estimated based on data from five time points using the serial longitudinal registration tool in SPM12 (Ashburner and Ridgway, 2012). Average images were then segmented into tissue classes using enhanced tissue probability maps (Lorio et al., 2016). However, the contrast of T1 images was not sufficient enough to segment deep gray matter structures, and these were classified as white matter. We excluded deep gray matter areas using the ICBM CerebraA atlas (http://nist.mni.mcgill.ca/?p=904). The approach we have applied relies on the registration of the average T1-weighted images (from each subject) to ICBM atlas (ICBM 2009a Nonlinear Symmetric). Once the nonlinear warps and transforms were obtained, we inversely warped the CerebrA atlas to structural space and subtracted deep gray matter structures (putamen $L / R$, pallidum $L / R$, thalamus $L / R$, lateral ventricle $L / R$, caudate $\mathrm{L} / \mathrm{R}$ ) from segmented white matter masks.

Diffusion MRI data were prepared for statistical analyses according to an established preprocessing pipeline (Ades-Aron et al., 2018). Images were denoised with a Marchenko-Pastur principal component analysis filter (Veraart et al., 2016), corrected for Gibbs ringing (Kellner et al., 2016), geometric EPI and motion distortion (FSL eddy; Andersson and Sotiropoulos, 2016), and B0 field inhomogeneities (Andersson et al., 2001). DTI metrics (FA, AD, and RD) were calculated from diffusion tensor estimated with weighted linear least squares (Veraart et al., 2013). Before performing voxel-based analysis on scalar diffusion maps, we created a study-specific group template from each individual's FA map at each time point [for visualization, see scheme_normalization.png file in the Open Science Framework (OSF) repository]. An initial within-subject template (rigid-affine spatial registration) was created with the "buildtemplateparallel" batch in ANTs (Avants et al., 2011). The resulting intersubject templates served as single-subject representatives in group spatial registration (rigid-affine and symmetric diffeomorphic registration) to the HCP1065 standard-space [Montreal Neurological Institute (MNI)] FA template with "antsMultivariateTemplateConstruction2" batch in ANTs (Avants et al., 2008). In this step, maps were upsampled to $1 \mathrm{~mm}^{3}$ isotropic voxel. Each individual's time point diffusion maps (FA, $\mathrm{AD}$, and $\mathrm{RD}$ ) were spatially registered to the study-specific group FA template in MNI space with priorly obtained registration matrices and deformation fields. In parallel, white matter average tissue maps derived from T1-weighted data were warped to the longitudinal intersubject FA template with transformations obtained from FA template registration to longitudinal T1 template. Finally, spatially registered DTI maps were smoothed by weighting with subjectspecific white matter probability maps (Lee et al., 2009) with a $4 \mathrm{~mm}$ isotropic kernel. Smoothing was performed within white matter masks to reduce partial volume effects. Additionally, we used an identical data processing strategy for TP0 and TP3 data selectively in the control and experimental groups.

Statistical analysis. Behavioral data were analyzed with repeatedmeasures (RM) ANOVAs: one-way with time (five time points) as a within-subject factor. Post hoc tests were calculated with Bonferroni correction for multiple comparisons. The Greenhouse-Geisser $F$ test and degrees of freedom correction were used for cases where sphericity assumptions were violated.

The FA maps were analyzed in two RM ANOVAs. First, braille group data (five time points) were analyzed in $5 \times 2$ repeated-measures ANOVA with time (TP0/TP1/TP2/TP3/TP4) as within-subject and reading hand (right/left) as between-subject factors, and an additional subject factor to account for the dependency of the acquired data. Since there was no significant effect of reading hand and no interaction between time and reading hand, the time effect was tested for pooled groups of right-handed and left-handed readers. The main effect of time contrast was thresholded at $p<0.001$ voxel-wise and $p<0.05$ familywise error (FWE) cluster corrected and used as an explicit mask for $2 \times 2$ ANOVA (braille-control) analysis to test for structural plasticity specific to braille reading training.

A $2 \times 2$ ANOVA with time (TP0/TP3) as within-subject and group (braille-control) as between-subject factors, and an additional subject factor was performed to investigate the interaction between group and time. The results are reported at a threshold of $p<0.001$ voxelwise, and were FWE cluster corrected for multiple comparisons to $p<0.05$. 
Additionally, we performed region of interest (ROI) analysis within spheres (radius $=4 \mathrm{~mm}$; to match the filter kernel size, corresponding to the smallest cluster size from either $2 \times 2$ ANOVA or $5 \times 2 \mathrm{RM}$ ANOVA) defined around peak $F$ values obtained in $5 \times 2$ RM ANOVA. Analysis was performed using linear mixed effect (LME) model followed by pairwise comparisons estimated marginal means implemented in nlme (Laird and Ware, 1982) and emmeans (Searle et al., 1980) packages, in R version 3.6.0 (R Development Core Team, 2019), respectively. We computed post-training-pretraining (TP3-TP0, $2 \times 2$ ANOVA) differences in DTI measures and entered them into two-sample $t$ tests with braille and passive control groups accordingly. All post hoc comparisons were Bonferroni corrected for the number of performed statistical tests, including time (if applicable) and diffusion metrics (FA, AD, and RD). Specifically, $p$ values in the models including two TPs were multiplied by $9-3$ (diffusion metrics) $\times$ three statistical tests (two one-sample $t$ tests measuring the change significance within each group and one twosample $t$ test representing the interaction), and in the models with five TPs $p$ values were multiplied by 30 (10 possible time comparisons $\times 3$ metrics). In addition to SEMs, error bars for plots (see Fig. 3) representing longitudinal data were adjusted to correctly reflect the variance in the within-subject design by removing between-subject differences accounted for in the repeated-measures statistical analyses (for details, see Cousineau, 2005; Morey, 2008). Both raw and normalized data as well as the R code used for transformations are available in the OSF project. Anatomical WM structures were labeled with "MRI Atlas of Human Brain Matter" (Oishi et al., 2010).

Finally, we calculated correlations between behavioral measures and FA with the rmcorr package (Bakdash and Marusich, 2017) in R version 3.6.0 (R Development Core Team, 2019), as it allows using all repeatedmeasures data points while not violating the assumption of the independence of observations. Confidence intervals (CIs) for correlation coefficients were calculated by bootstrap method with 100 resamples.

Data availability. Source data used to create all figures along with the code for data processing and statistical analysis are available at OSF project directory: https://osf.io/tqh6f/?view_only= f7cc63c3e87e4449b09d7953e67dd23f. ROI-level data and codes to perform statistical analysis are provided in the $\mathrm{R}$ project code. Preprocessed MRI data that support the findings of this study are available on request from the corresponding authors. The data are not publicly available because of privacy protection regulations.

\section{Results}

Before training, of 26 subjects, 1 subject was able to read three words, 1 subject read two words, and 2 subjects read one word per minute (group mean $=0.31$ words; $\mathrm{SD}=0.11$; range, $0-3$ words). At the end of the training, subjects were able to read approximately eight words per minute (mean =8.04 words; $\mathrm{SD}=4.13$; range, $1-17$ words). The ANOVA showed a significant effect of time $\left(F_{(4,96)}=60.73 ; p<0.0001\right)$, indicating reading improvement over time. Post hoc tests between adjacent TP pairs showed significant increase in reading speed between TP0 and $\mathrm{TP} 1\left(t_{(26)}=7.2, p_{\text {corr }}<0.0001\right), \mathrm{TP} 1$ and TP2 $\left(t_{(26)}=6.4, p_{\text {corr }}<\right.$ $0.0001)$, trend-level increase between TP2 and TP3 $\left(t_{(26)}=2.6\right.$, $\left.p_{\text {corr }}=0.066\right)$, as well as a significant decrease in follow-up (TP4) compared with post-training $(\mathrm{TP} 3)$ test $\left(t_{(26)}=4.8, p_{\text {corr }}<0.001\right.$; Fig. 1B).

\section{Pretraining-post-training effects of braille training (comparison with control group)}

Effects specific to tactile training were tested in the interaction between group (braille-control) and time (TP0 and TP3; Fig. 2). The analysis revealed FA increases in left postcentral gyrus (PoG)-WM L (cluster extent $=115 ; F=48.83$; MNI $=[-13,-29$, 53]) and left middle/lateral occipital (O2)-WM L (cluster extent $=112 ; F=34.97 ; \mathrm{MNI}=[-22,-83,-1])$. Moreover, there was a significant FA decrease in the right posterior part of the internal capsule (pic R; cluster extent $=223 ; F=22.37$; MNI = $[21,-12,3])$. To further characterize the observed alterations in $\mathrm{FA}$, we analyzed $\mathrm{AD}$ and $\mathrm{RD}$ within each above region of interest. $t$ Tests were conducted on diffusion measures separately, and $p$ values were adjusted with Bonferroni correction within each ROI. The significant FA post-training difference between braille and control subjects $\left(t_{(47.98)}=-6.388 ; p_{\text {corr }}<0.001\right.$; with significant increase in braille group: $\left.t_{(24)}=7.081 ; p_{\text {corr }}<0.001\right)$ in PoG-WM L was accompanied by a significant interaction in RD $\left(t_{(47.97)}=3.177 ; p_{\text {corr }}<0.05\right)$ driven by a decrease of the measure in the braille group after training $\left(t_{(24)}=-4.064 ; p_{\text {corr }}<0.01\right.$ ), while no significant differences between groups in $\mathrm{AD}$ were found $\left(t_{(44.75)}=-2.262 ; p_{\text {corr }}>0.05\right)$. For O2-WM L, there was a significant group difference in FA $\left(t_{(46.69)}=-5.495\right.$; $p_{\text {corr }}<$ $0.0001)$. More specifically, FA increase $\left(t_{(24)}=5.116 ; p_{\text {corr }}<\right.$ 0.001 ) was followed by a significant change in RD and a nonsignificant change in $\mathrm{AD}$ in the braille group $\left(t_{(24)}=-3.176 /\right.$ $p_{\text {corr }}<0.05$ and $t_{(24)}=2.214 / p_{\text {corr }}>0.05$, respectively) . Additionally, there was a nonsignificant group difference $\left(t_{(47.95)}=2.035 ; p_{\text {corr }}>0.05\right)$ in $\mathrm{RD}$, whereas a significant pretraining-post-training group difference in $\mathrm{AD}\left(t_{(47.98)}=\right.$ $\left.-3.842 ; p_{\text {corr }}<0.001\right)$, driven by a significant decrease in the control group $\left(t_{(24)}=-3.230 ; p_{\text {corr }}<0.05\right)$, was detected. The significant FA post-training decrease in the braille group compared with control subjects $\left(t_{(47.99)}=4.529 ; p_{\text {corr }}<\right.$ 0.001 ; with a significant decrease within the braille group: $t_{(24)}=-5.228$; $\left.p_{\text {corr }}<0.001\right)$ in pic $\mathrm{R}$ was followed by significant group difference in $\mathrm{RD}\left(t_{(45.98)}=-4.464 ; p_{\text {corr }}<0.001\right)$, with RD increase in the braille group $\left(t_{(24)}=4.385 ; p_{\text {corr }}<\right.$ $0.01)$. However, there were no significant differences either between or within the braille and control groups (group interaction, $t_{(47.63)}=-1.044$; braille group, $t_{(24)}=0.025$; control group, $t_{(24)}=-1.519$; all $p_{\text {corr }}$ values $\left.>0.05\right)$ in $\mathrm{AD}$.

\section{Time course of braille training effects}

Aiming to understand the dynamics of training changes, we analyzed diffusion measures in specific ROIs. ROIs obtained from braille group-control group interaction (Fig. 2) were extracted from longitudinal (five TPs) braille group analysis.

The time courses of each region and metrics are depicted in Figure 3. The LME model was applied for each diffusion measure separately, and $p$ values were adjusted with Bonferroni correction within each ROI. The LME model with Bonferroni correction revealed significant time effects for every ROI and diffusion measure (PoG-WM L: FA: $t_{(100)}=10.702 ; p_{\text {corr }}<0.0001$; RD: $t_{(100)}=$ 8.471; $p_{\text {corr }}<0.0001$; AD: $t_{(100)}=4.671, p_{\text {corr }}<0.01$; O2-WM L: FA: $t_{(100)}=12.286 ; p_{\text {corr }}<0.0001 ; \mathrm{RD}: t_{(100)}=6.052 ; p_{\text {corr }}<$ 0.001 ; pic R: FA: $t_{(100)}=15.719$; $p_{\text {corr }}<0.0001$; RD: $t_{(100)}=$ 11.208 ; $\left.p_{\text {corr }}<0.0001\right)$ in addition to AD in O2-WM L and in pic $\mathrm{R}\left(t_{(100)}=0.636\right.$ and $t_{(100)}=2.299$, respectively; all $p_{\text {corr }}$ values $>0.05)$. Post hoc comparisons with Bonferroni correction were evaluated only for statistically significant measures within each ROI after the LME model analysis. For PoG-WM L, there was a linear-like increase of FA over the whole training period with pretraining-post-training (TP0TP3) significance of $t_{(100)}=-5.561$ and $p_{\text {corr }}<0.0001$. In the follow-up, FA significantly dropped after the end of training (TP4-TP3: $\left.t_{(100)}=5.145 ; p_{\text {corr }}<0.0001\right)$ and reached baseline level. The analysis of RD in PoG-WM L revealed significant decreases over the first 6 months (TP2-TP0) of training (RD: $\left.t_{(100)}=4.883 ; p_{\text {corr }}<0.0001\right)$. RD and AD increased over the TP2-TP4 period $\left(t_{(100)}=-4.287 ; p_{\text {corr }}<0.001\right)$ and TP2-TP3 period $\left(t_{(100)}=-4.109 ; p_{\text {corr }}<0.001\right)$, respectively, 


\section{Postcentral Gyrus WM L}
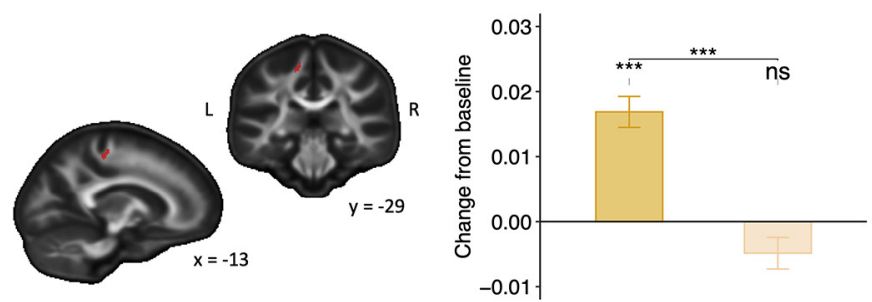

Middle/Lateral Occipital Gyrus WM L
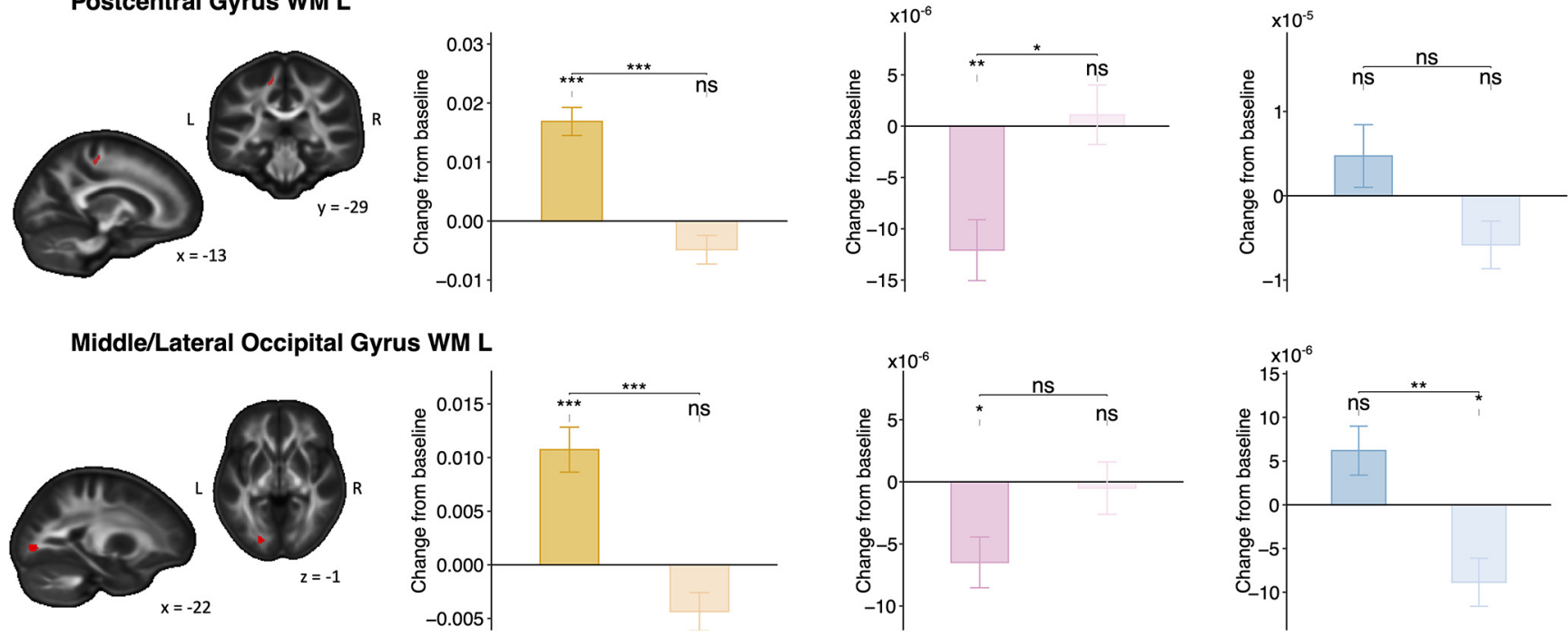

Posterior part of internal capsule R
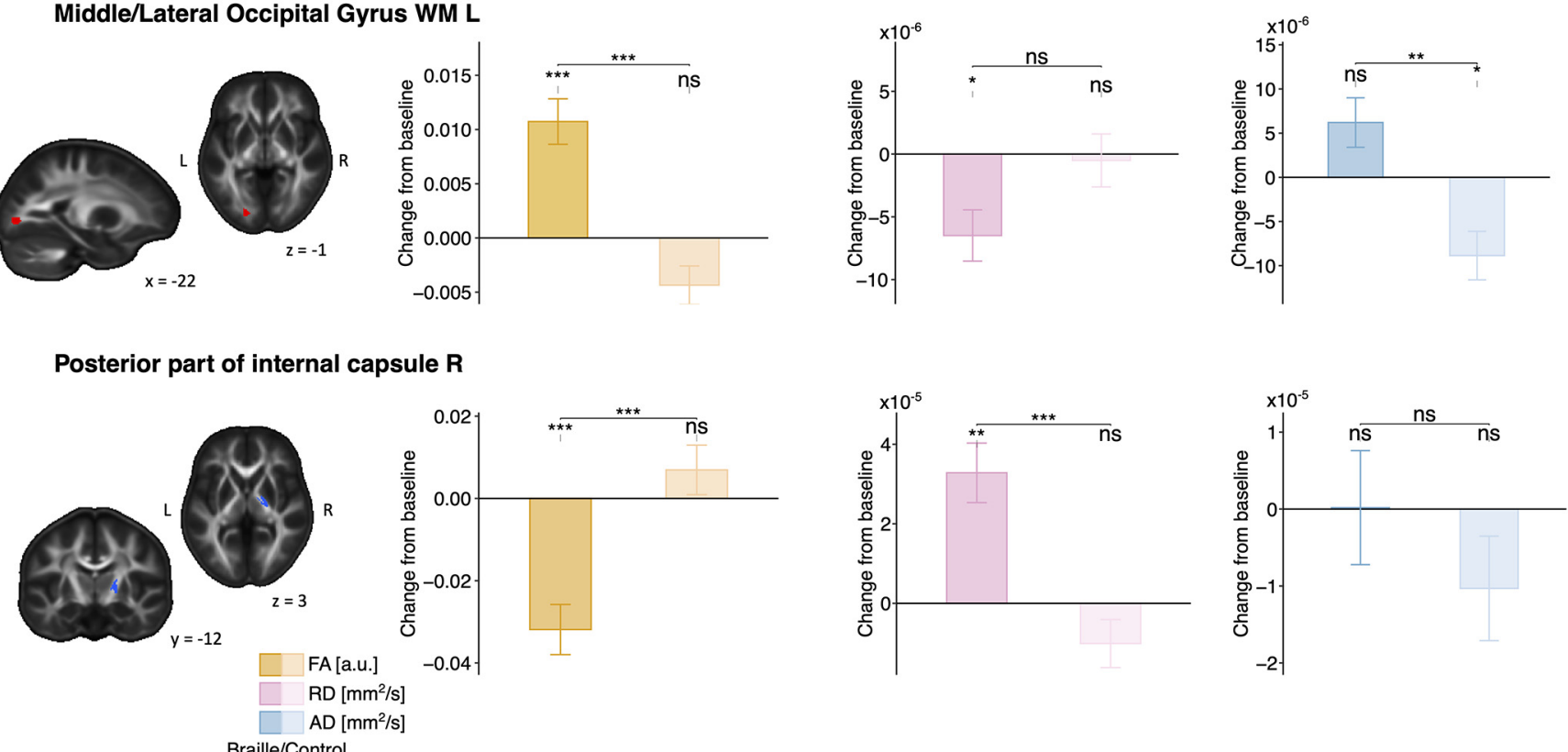

Figure 2. Areas specific to braille training effects overlaid on the group-specific FA-MNI template. Voxel clusters of increased or decreased FA are denoted by red and blue, respectively (left). Time (TP3 - TP0) $\times$ group (braille - control) interactions: braille group (post-training-pretraining) $>$ control group (post-training-pretraining) comparison evaluated for FA, AD, and RD measures. Asterisks between bars indicate significant group $\times$ time interactions: ${ }^{*} p<0.05,{ }^{* *} p<0.01,{ }^{* * *} p<0.001$, Bonferroni corrected (right). ns, Not significant, a.u., arbitrary units. $X$, $\mathrm{Y}$ and $\mathrm{Z}$ represent coordinates in MNI space. Error bars represent SEMs adjusted for within-subject designs (Morey, 2008).

to reach the onset level after training in the follow-up period (TP4). In the $\mathrm{O} 2-\mathrm{WM} \mathrm{L}$, we observed significant FA increase between the third and sixth month (TP1-TP2) of training $\left(t_{(100)}=-3.795\right.$; $p_{\text {corr }}<0.01$ ), and that change remained significant till the end of the braille course (TP1-TP3: $t_{(100)}=-4.863$; $\left.p_{\text {corr }}<0.0001\right)$. Similar to the PoG-WM L FA time course, FA decreased after TP3 and reached the baseline in the follow-up (TP4: $t_{(100)}=4.646 ; p_{\text {corr }}=$ $0.0001)$. The $\mathrm{RD}$ time course for vision-associated white matter presents a reversed pattern: $\mathrm{RD}$ decreased in the TP1-TP2 time window $\left(t_{(100)}=3.130 ; p_{\text {corr }}<0.05\right)$, and this change remained significant in TP0-TP3 comparison $\left(t_{(100)}=3.266 ; p_{\text {corr }}<0.05\right)$. Both measures reached the baseline in the follow-up. For motor-associated areas, the most significant reorganization occurred in the TP1TP2 time window denoted by FA decrease $\left(t_{(100)}=3.682 ; p_{\text {corr }}<\right.$ 0.01 ). The FA change remained significant till the course end (TP3TP0: $\left.t_{(100)}=5.711 ; p_{\text {corr }}<0.0001\right)$ and increased to reach the baseline in the follow-up. For the right posterior part of internal capsule, post hoc analysis detected a significant RD increase between TP1 and $\operatorname{TP} 3\left(t_{(100)}=-4.297 ; p_{\text {corr }}<0.001\right)$ and a decrease in TP4 $\left(t_{(100)}=\right.$ 6.238; $\left.p_{\text {corr }}<0.0001\right)$. There were no significant changes in $\mathrm{AD}$ detected either in the O2-WM L or in the pic R across the study duration and follow-up.

\section{Brain-behavior correlations during learning}

To test the association between structural plasticity and braille reading skills, we used repeated-measures correlations (Bakdash and Marusich, 2017) between tactile reading speed (measured in words per minute) and absolute values of FA at each TP extracted from regions reported in Figure 2. Since in TP0 subjects were naive to tactile reading, only data from TP1-TP4 were included in the analysis. Correlation analysis showed a significant positive relationship of tactile reading speed with FA in the motor area (PoG-WM L: $r_{(77)}=0.35 ; p<0.01$; CI, 0.153-0.551; Fig. 4, left) and in the visual cortex (O2-WM L: $r_{(77)}=0.47$; $p<0.0001$; CI, 0.330-0.604; Fig. 4, middle). Negative relationship with FA was observed in the right posterior part of the internal capsule $\left(r_{(77)}=-0.45 ; p<0.0001\right.$; CI, -0.575 to -0.284 ; Fig. 4, right).

\section{White matter reorganization over time (braille group)}

To localize changes that were not specific to the interaction between TP0 and TP3 and the control group, we tested for the main effect of time across the whole brain. We show changes in motor-related (bilateral superior corona radiata, bilateral posterior limb of internal capsule, precentral and superior frontal gyri), visual-related (middle/lateral occipital gyrus), and language-related (supramarginal gyrus) white matter areas (Fig. 5, Table 1). There was no time $\times$ reading hand interaction. As a consequence, the time effect was tested for pooled groups of right-handed and left-handed readers. However, since these results are not compared with the control group because of the limitation of our design, we did not explore the detailed time 


\section{Postcentral Gyrus WM L}

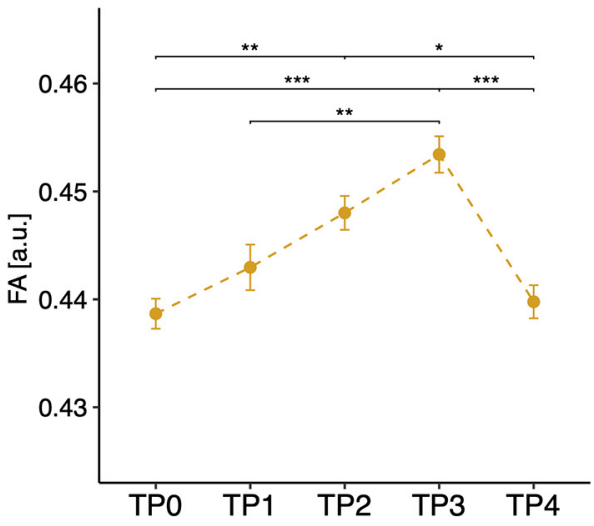

\section{Middle/Occipital Gyrus WM L}

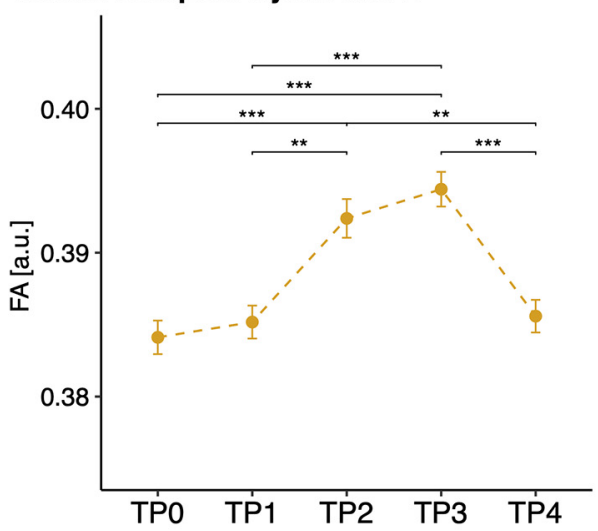

\section{Posterior part of internal capsule $\mathbf{R}$}

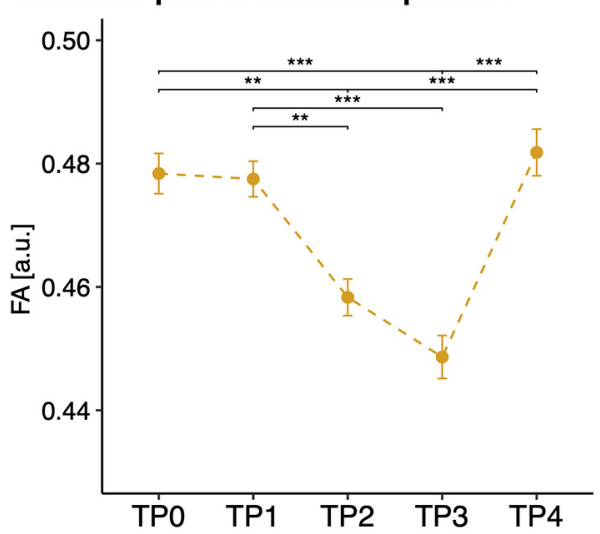

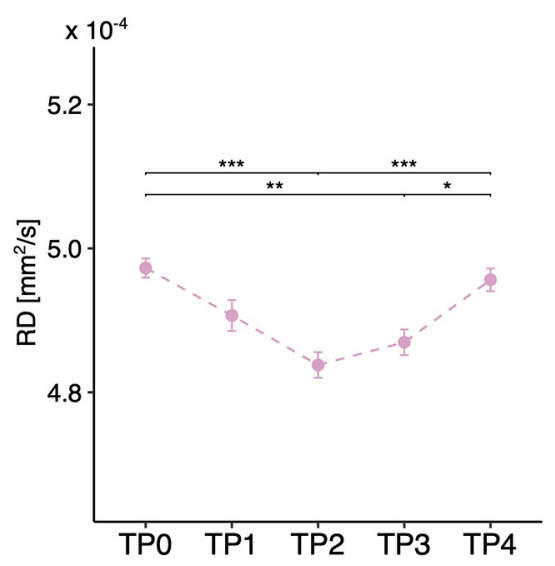
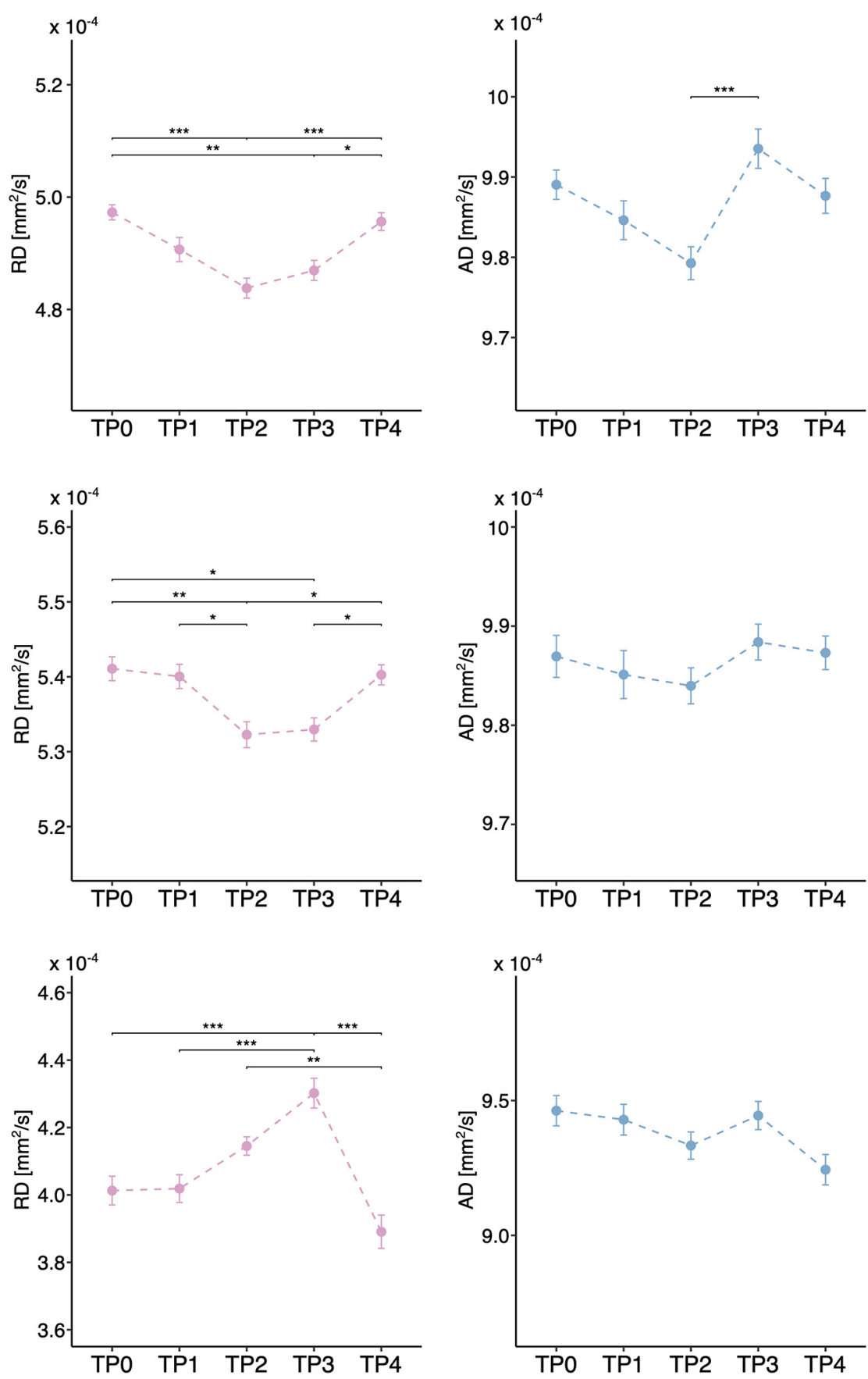

Figure 3. Region of interest analyses for braille-control interaction areas: detailed time courses of structural plasticity in the braille group during training. Error bars represent SEMs adjusted for within-subject designs (Morey, 2008). ${ }^{*} p<0.05,{ }^{* *} p<0.01,{ }^{* * *} p<0.001$, Bonferroni corrected.

courses. The specificity of the observed changes should be analyzed in future studies.

\section{Discussion}

In this study, we monitored WM microstructure reorganization in sighted subjects exposed to intensive training in tactile braille reading. We show that learning-specific WM plasticity occurs both intramodally and cross-modally, which was reflected by FA increases in somatosensory and visual cortex, respectively. The observed changes followed distinct time courses, with visual cortex cross-modal plasticity occurring only after achieving a proficiency level that allows the reading of braille words (Fig. 3). WM reorganization observed in these areas returned to baseline after the cessation of learning. We provide additional evidence that FA changes in the visual and somatosensory cortices as well as the subcortical part of the motor tracts are behaviorally relevant to tactile reading (Fig. 4). Together, these results demonstrate that brain plasticity is a highly dynamic process modulated by the introduction of novel experiences.

Braille reading involves first and foremost the evaluation of novel tactile input. This input is present from the very beginning of learning in the form of basic tactile discrimination and the introduction of single letters followed by more complex stimuli until the end of training (Fig. 1A). In our data, this gradient was paralleled by $\mathrm{FA}$ increases together with $\mathrm{RD}$ decreases in the 

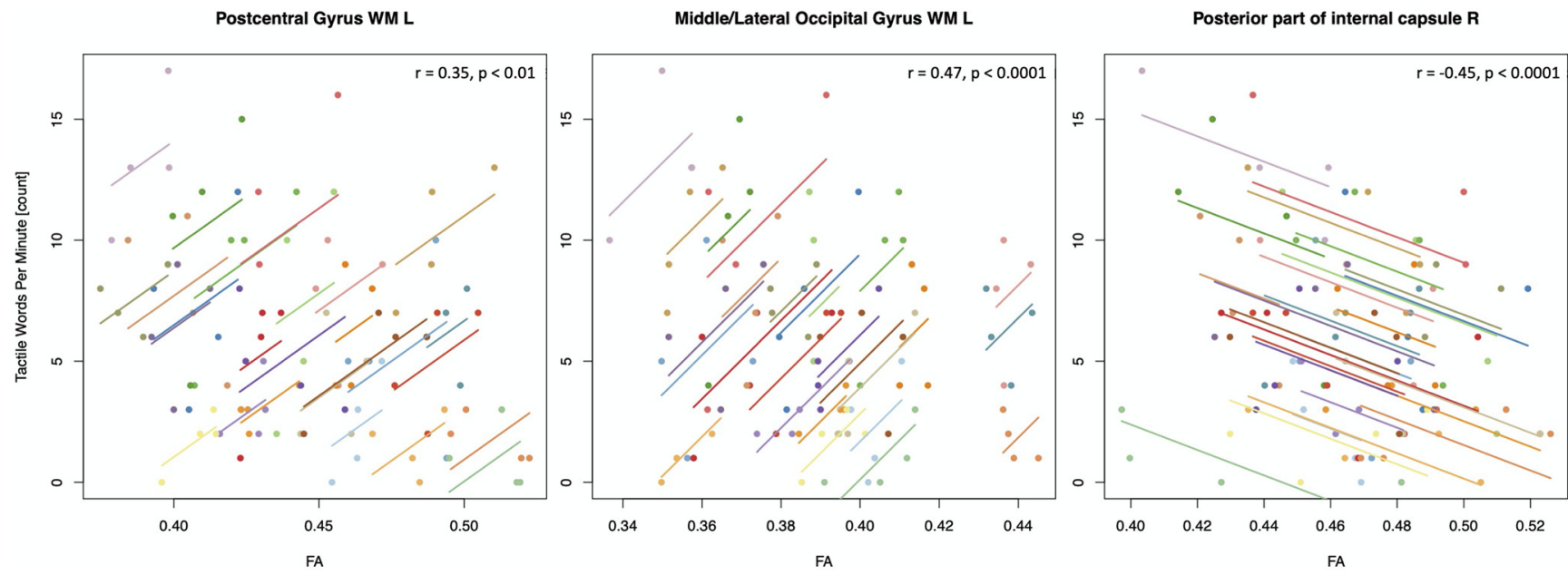

Figure 4. Brain-behavior correlations: relations between FA and braille reading speed [measured by tactile words per minute (tWPM)]. A set of four same-color dots represents separate observations of FA and tWPM from the same participant (alongside a color-matched regression line fit). Analyses were performed with repeated-measurements correlations (Bakdash and Marusich, 2017) using data from TP1 to TP4.
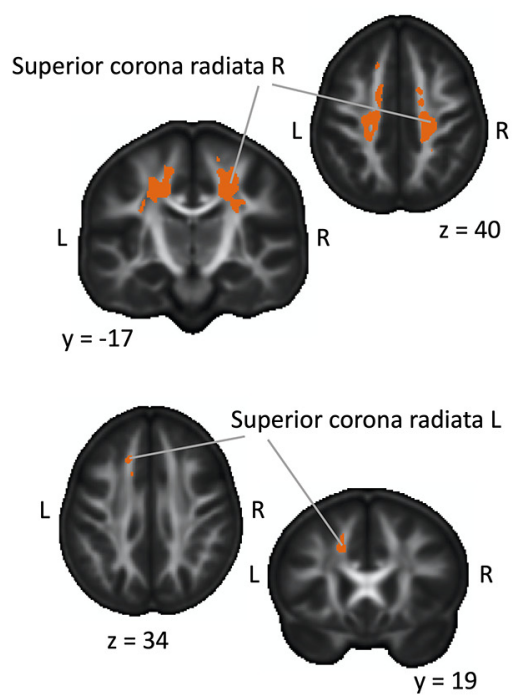
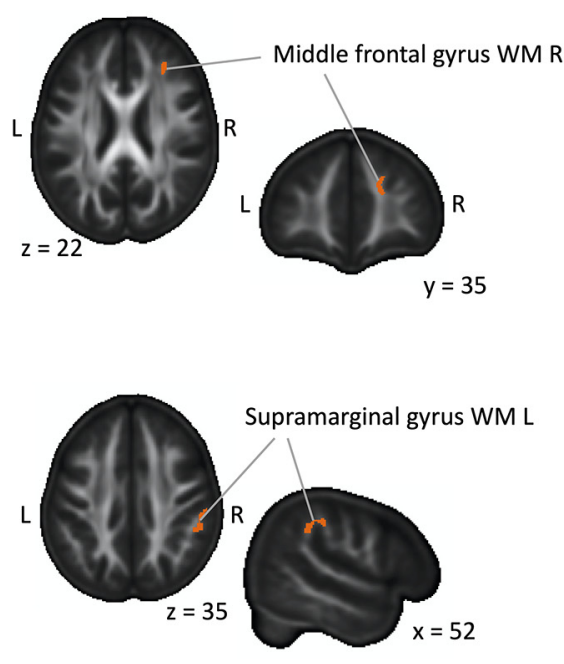
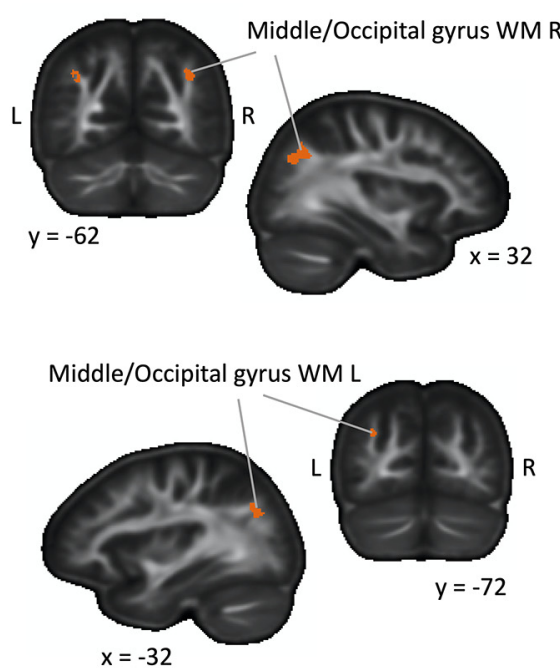

Figure 5. Main effect of time point: white matter FA changes in the braille readers group. Anatomical WM structures were labeled with MRI Atlas of Human Brain Matter (0ishi et al., 2010; $p=0.05$, FWEc $\left.=213, p_{\text {unc }}=0.001\right) . X, Y$ and $Z$ represent coordinates in MNI space.

Table 1. White matter FA changes in the experimental group for all time points

\begin{tabular}{lrrrrrr}
\hline & & & \multicolumn{3}{c}{ MNI coordinates } \\
\cline { 5 - 7 } Region & Extent & & $F$ value & $x$ & \multicolumn{1}{c}{$y$} & \multicolumn{1}{c}{$z$} \\
\hline Posterior limb of internal capsule R & 464 & 17.20 & 21 & -12 & 3 \\
Precentral gyrus WM L & 4283 & 14.17 & -10 & -24 & 58 \\
Superior corona radiata R & 3727 & 13.76 & 19 & -17 & 40 \\
Supramarginal gyrus WM R & 321 & 12.36 & 52 & -33 & 35 \\
Middle/occipital gyrus WM L & 312 & 11.37 & -21 & -84 & -2 \\
Posterior corona radiata R & 302 & 10.75 & 22 & -32 & 23 \\
Middle/occipital gyrus WM R & 290 & 9.29 & 32 & -62 & 33 \\
Superior corona radiata L & 213 & 9.06 & -16 & 19 & 34 \\
Superior frontal gyrus WM R & 377 & 8.84 & 17 & -6 & 42 \\
Middle/occipital gyrus WM L & 296 & 8.80 & -32 & -72 & 27 \\
Middle frontal gyrus WM R & 235 & 8.45 & 27 & 35 & 22 \\
Posterior limb of internal capsule L & 535 & 7.77 & -22 & -15 & 5 \\
\hline
\end{tabular}

Anatomical WM structures were labeled using the MRI Atlas of Human Brain Matter (0ishi et al., 2010; $p=0.05 ;$ FWEC $=213, p_{\text {unc }}=0.001$, MNI peak $F$ values $)$. somatosensory WM. Studies based on animal models indicated that simultaneous alterations in these two metrics could be driven by myelination (Beaulieu, 2002; Song et al., 2005; Sampaio-Baptista et al., 2013, 2020), which was suggested as central to learning-induced neuroplasticity (Fields, 2010, 2015; Heath et al., 2018). Such intramodal reorganization within the trained sense seems intuitive and was observed in other studies involving functional and structural changes in tactile experiences including short-term tactile reading training (Debowska et al., 2016), somatotopy remapping (Kolasinski et al., 2016), somatosensory stimulation (Schmidt-Wilcke et al., 2018), or perceptual learning (Pleger et al., 2003). Recently, we also showed a similar pattern of gradual anatomic changes of sensory cortices in the gray matter of sighted braille learners (Matuszewski et al., 2021). Additionally, FA of the somatosensory WM was correlated with the acquired tactile reading speed (Fig. 4, left). The exact role of the somatosensory cortex in tactile reading has been debated, with some studies observing its functional and 
structural remapping (Debowska et al., 2016), and others not (Siuda-Krzywicka et al., 2016; Bola et al., 2017). Our results showed that gradual microstructural reorganization in the somatosensory WM might be related to simple exposure to novel tactile stimuli further linked to the progress in braille reading. These novel stimuli, after basic evaluation, require fine-grained spatial analysis of the characters occurring in the visual cortex (Saito et al., 2006; Merabet et al., 2008; Stilla and Sathian, 2008; Sathian et al., 2011; Bola et al., 2019).

In previous research, involvement of the visual cortex in the processing of nonvisual information was reported both with specific training (Saito et al., 2006; Zangenehpour and Zatorre, 2010) and without specific training (Amedi et al., 2001; Sathian et al., 2011; Eck et al., 2013, 2016). Visual cortex anatomy can also undergo reorganization by various types of training, including color naming (Kwok et al., 2011), juggling (Draganski et al., 2004; Scholz et al., 2009), mirror reading (Ilg et al., 2008), or 9 months of tactile reading training (Bola et al., 2017). Here we show that, contrary to continuous microstructural reorganization in the somatosensory cortex, FA increases (mirrored by significant RD decreases) in the visual WM occur after 3 months of training, when subjects are progressing from reading single letters to reading words (Fig. $1 A$, three middle panels). Furthermore, FA of the visual WM was positively correlated with tactile reading speed (Fig. 4, middle). These results indicate that the temporal dynamics of microstructural plasticity might be modulated by the nature of computational demands. Since tactile reading assimilates novel sensory input into an existing language network, FA changes found in the middle/occipital gyrus could reflect improved connectivity between areas participating in detailed spatial analysis of haptic stimuli and high-level visual or language networks via inferior fronto-occipital or longitudinal fasciculus. Such neuroplastic changes would occur only when braille became linguistically meaningful. This hierarchy and information flow between visual areas during tactile reading was verified in our additional study incorporating chronometric TMS (Bola et al., 2019).

Tactile reading also carries an important motor component, as it relies on fine-tuned hand and finger movements required for proper braille character identification. In our study, this motor domain was reflected by significant FA changes in the posterior limb of internal capsule, which contains corticospinal tracts. Counterintuitively, the temporal dynamic analysis indicated decreases in FA during training, followed by return to baseline in the follow-up scan (Fig. 3, bottom). Moreover, FA in this region was negatively correlated with the tactile reading speed. This pattern of changes is highly unlikely to be caused by the axon demyelination observed (i.e., in aging; Lebel et al., 2012; Sexton et al., 2014). Multiple reviews showed that FA-inferred information about microstructural anisotropy is significantly affected by underlying tissue characteristics, particularly crossing fibers (Tuch et al., 2002; Jbabdi et al., 2010; Vos et al., 2011). In that view, FA decreases could be observed on the voxel level even if the task-relevant part of the fibers would increase its FA. Similar decreases during learning were reported in previous studies (Taubert et al., 2010). Specifically, the posterior limb of internal capsule hosts sensory fibers of the corticospinal tract leading to motor areas as well as tracts of optic radiation. Additionally, one recent study also demonstrated possible homunculus-like organization of fibers in PIC (Qian and Tan, 2017). Therefore, selective FA increase in hand fibers could be masked by the underlying WM microstructure.
Finally, exploratory analysis provided preliminary evidence that during training changes over time also appear in other regions (Table 1), which could be broadly classified as motor, visual, or language related. In these regions, the reorganization might have occurred with a different timeline, either before participants reached peak performance or in the follow-up; therefore, it was not visible in the interaction with the passive control group. Thus, its relevance for braille reading learning should be verified in future studies.

We did not observe any training-specific structural changes located in the reading network. However, as braille is an alphabet presented in an atypical sensory modality, only the nature of input is novel, while it enables access to an already known (Polish) language. This explains why anatomic changes are observed in sensory regions and not in the higher-order parts of the reading network located in the temporal or frontal lobe. This could be indirectly supported by various studies reporting left inferior frontal gyrus anatomic reorganization in bilingual individuals or during the acquisition of a second language (Li et al., 2014; Banaszkiewicz et al., 2021).

Our results are in line with the view that WM demonstrates highly dynamic, experience-dependent plasticity during learning (Sampaio-Baptista and Johansen-Berg, 2017). In our study, FA in the areas undergoing training-specific reorganization returned to baseline values (Fig. 3) during a follow-up scan (TP4) after the withdrawal from tactile reading. This finding is in line with the supply-demand model of plasticity (Lövdén et al., 2010). In this view, experience-dependent brain structure changes are assumed to adapt to demands introduced by novel experiences. Specifically, this process is observed in continuous increases in a given brain structure metric (i.e., volumetric change) until the demands for optimal processing are met. Similar patterns of WM properties were reported by Glasø de Lange et al. (2018), where older adults followed a 40 week program consisting of alternating memory training and rest periods. In their study, changes in FA and MD approximately followed an S-shaped curve, with increases during training and retractions during resting periods.

Several key limitations of this study should be discussed. First, although DTI provides markers sensitive to microstructural tissue reorganization, its metrics are not specific to biologically interpretable tissue properties (Lerch et al., 2017). Furthermore, DTI provides only a rough characterization of the underlying structure, which captures the most prominent features at low $b$ values (Novikov et al., 2018). Multiple biophysical diffusion models [e.g., NODDI (neurite orientation dispersion and density imaging) or CHARMED (composite hindered and restricted model of diffusion)] allowing more fine-tuned analysis of the WM microstructure have been proposed to tackle this issue (for review, see Panagiotaki et al., 2012; Nilsson et al., 2013). However, biophysical modeling requires a priori knowledge of the tissue characteristics to appropriately estimate different compartments (e.g., sticks used to describe fibers) and to accurately derive scalar metrics. Additionally, inclusion of the quantitative MRI recently showed distinctive profiles of reading (and math) network tract sub-bundles (Grotheer et al., 2019). Therefore, using advanced multicontrast and optimized MRI acquisitions would contribute to a deeper understanding of the tissue changes during braille learning by mapping (i.e., crossing fibers, neurite densities, or myelin water fractions). Second, test-retest reliability of the diffusion metrics over time would 
provide useful information regarding the stability of MRI measurements. However, in this case shorter time scales of minutes (Koller et al., 2021; Veraart et al., 2021) or days (Landman et al., 2011 Luque Laguna et al., 2020), instead of several months, are needed. Last, inclusion of the passive control group allowed us to measure the specificity of the experience-induced neuroplasticity. However, our design incorporated control scans only before (TP0) and after (TP3) training. While this approach allowed us to capture the specificity of cumulated learning effects, it limited the conclusions about the detailed time course of WM reorganization.

In conclusion, with a unique longitudinal design including multiple time points we provide novel perspectives into the dynamics of experience-induced cross-modal neuroplasticity. We demonstrate that in sighted adults, behaviorally relevant microstructural properties of WM are modulated by learning with regionally specific time courses. Additionally, observed changes returned to baseline after the cessation of learning, supporting the view that WM changes, possibly driven by myelin plasticity, follow the supply-demand pattern of neuroplasticity.

\section{References}

Ades-Aron B, Veraart J, Kochunov P, McGuire S, Sherman P, Kellner E, Novikov DS, Fieremans E (2018) Evaluation of the accuracy and precision of the diffusion parameter EStImation with Gibbs and NoisE removal pipeline. Neuroimage 183:532-543.

Amedi A, Malach R, Hendler T, Peled S, Zohary E (2001) Visuo-haptic object-related activation in the ventral visual pathway. Nat Neurosci 4:324-330.

Andersson JLR, Sotiropoulos SN (2016) An integrated approach to correction for off-resonance effects and subject movement in diffusion MR imaging. Neuroimage 125:1063-1078.

Andersson JL, Hutton C, Ashburner J, Turner R, Friston K (2001) Modeling geometric deformations in EPI time series. Neuroimage 13:903-919.

Ashburner J, Ridgway GR (2012) Symmetric diffeomorphic modeling of longitudinal structural MRI. Front Neurosci 6:197.

Avants BB, Epstein C, Grossman M, Gee J (2008) Symmetric diffeomorphic image registration with cross-correlation: evaluating automated labeling of elderly and neurodegenerative brain. Med Image Anal 12:26-41.

Avants BB, Tustison NJ, Song G, Cook PA, Klein A, Gee JC (2011) A reproducible evaluation of ANTs similarity metric performance in brain image registration. Neuroimage 54:2033-2044.

Bakdash JZ, Marusich LR (2017) Repeated measures correlation. Front Psychol 8:456.

Banaszkiewicz A, Matuszewski J, Bola Ł, Szczepanik M, Kossowski B, Rutkowski P, Szwed M, Emmorey K, Jednoróg K, Marchewka A (2021) Multimodal imaging of brain reorganization in hearing late learners of sign language. Hum Brain Mapp 42:384-397.

Basser PJ, Mattiello J, LeBihan D (1994) MR diffusion tensor spectroscopy and imaging. Biophys J 66:259-267.

Beaulieu C (2002) The basis of anisotropic water diffusion in the nervous system - a technical review. NMR Biomed 15:435-455.

Bola E, Siuda-Krzywicka K, Paplińska M, Sumera E, Hańczur P, Szwed M (2016) Braille in the sighted: teaching tactile reading to sighted adults. PLoS One 11:e0155394.

Bola Ł, Siuda-Krzywicka K, Paplińska M, Sumera E, Zimmermann M, Jednoróg K, Marchewka A, Szwed M (2017) Structural reorganization of the early visual cortex following braille training in sighted adults. Sci Rep $7: 17448$.

Bola $Ł$, Matuszewski J, Szczepanik M, Droździel D, Sliwinska MW, Paplińska M, Jednoróg K, Szwed M, Marchewka A (2019) Functional hierarchy for tactile processing in the visual cortex of sighted adults. Neuroimage 202:116084.

Caeyenberghs K, Metzler-Baddeley C, Foley S, Jones DK (2016) Dynamics of the human structural connectome underlying working memory training. J Neurosci 36:4056-4066.

Cousineau D (2005) Confidence intervals in within-subject designs: a simpler solution to Loftus and Masson's method. Tutor Quant Methods Psychol $1: 42-45$.
Debowska W, Wolak T, Nowicka A, Kozak A, Szwed M, Kossut M (2016) Functional and structural neuroplasticity induced by short-term tactile training based on braille reading. Front Neurosci 10:460.

Draganski B, Gaser C, Busch V, Schuierer G, Bogdahn U, May A (2004) Changes in grey, matter induced by training. Nature 427:311-312.

Eck J, Kaas AL, Goebel R (2013) Crossmodal interactions of haptic and visual texture information in early sensory cortex. Neuroimage 75:123-135.

Eck J, Kaas AL, Mulders JL, Hausfeld L, Kourtzi Z, Goebel R (2016) The effect of task instruction on haptic texture processing: the neural underpinning of roughness and spatial density perception. Cereb Cortex 26:384-401.

Fields RD (2010) Neuroscience. Change in the brain's white matter. Science 330:768-769.

Fields RD (2015) A new mechanism of nervous system plasticity: activity-dependent myelination. Nat Rev Neurosci 16:756-767.

Glasø de Lange A-M, Sjøli Bråthen AC, Rohani DA, Fjell AM, Walhovd KB (2018) The temporal dynamics of brain plasticity in aging. Cereb Cortex 28:1857-1865.

Grotheer M, Zhen Z, Lerma-Usabiaga G, Grill-Spector K (2019) Separate lanes for adding and reading in the white matter highways of the human brain. Nat Commun 10:3675.

Heath F, Hurley SA, Johansen-Berg H, Sampaio-Baptista C (2018) Advances in noninvasive myelin imaging. Dev Neurobiol 78:136-151.

Ilg R, Wohlschläger AM, Gaser C, Liebau Y, Dauner R, Wöller A, Zimmer C, Zihl J, Mühlau M (2008) Gray matter increase induced by practice correlates with task-specific activation: a combined functional and morphometric magnetic resonance imaging study. J Neurosci 28:4210-4215.

Jbabdi S, Behrens TE, Smith SM (2010) Crossing fibres in tract-based spatial statistics. Neuroimage 49:249-256.

Jbabdi S, Sotiropoulos SN, Haber SN, Van Essen DC, Behrens TE (2015) Measuring macroscopic brain connections in vivo. Nat Neurosci 18:1546-1555.

Koller K, Rudrapatna U, Chamberland M, Raven EP, Parker GD, Tax CMW, Drakesmith M, Fasano F, Owen D, Hughes G, Charron C, Evans CJ, Jones DK (2021) MICRA: microstructural image compilation with repeated acquisitions. Neuroimage 225:117406.

Kellner E, Dhital B, Kiselev VG, Reisert M (2016) Gibbs-ringing artifact removal based on local subvoxel-shifts. Magn Reson Med 76:1574-1581.

Kolasinski J, Makin TR, Jbabdi S, Clare XS, Stagg CJ, Johansen-Berg H (2016) Investigating the stability of fine-grain digit somatotopy in individual human participants. J Neurosci 36:1113-1127.

Kwok V, Niu Z, Kay P, Zhou K, Mo L, Jin Z, So KF, Tan LH (2011) Learning new color names produces rapid increase in gray matter in the intact adult human cortex. Proc Natl Acad Sci U S A 108:6686-6688.

Laird NM, Ware JH (1982) Random-effects models for longitudinal data. Biometrics 38:963-974.

Landman BA, Huang AJ, Gifford A, Vikram DS, Lim IAL, Farrell JAD, Bogovic JA, Hua J, Chen M, Jarso S, Smith SA, Joel S, Mori S, Pekar JJ, Barker PB, Prince JL, van Zijl PCM (2011) Multi-parametric neuroimaging reproducibility: a 3-T resource study. Neuroimage 54:2854-2866.

Lebel C, Gee M, Camicioli R, Wieler M, Martin W, Beaulieu C (2012) Diffusion tensor imaging of white matter tract evolution over the lifespan. Neuroimage 60:340-352.

Lebel C, Treit S, Beaulieu C (2019) A review of diffusion MRI of typical white matter development from early childhood to young adulthood. NMR Biomed 32:e3778.

Lee JE, Chung MK, Lazar M, DuBray MB, Kim J, Bigler ED, Lainhart JE, Alexander AL (2009) A study of diffusion tensor imaging by tissue-specific, smoothing-compensated voxel-based analysis. Neuroimage 44:870883.

Lerch JP, Van Der Kouwe AJW, Raznahan A, Paus T, Johansen-Berg H, Miller KL, Smith SM, Fischl B Stamatios and (2017) Studying neuroanatomy using MRI. Nat Neurosci 20:314-326.

Li P, Legault J, Litcofsky KA (2014) Neuroplasticity as a function of second language learning: anatomical changes in the human brain. Cortex 58:301-324.

Lorio S, Fresard S, Adaszewski S, Kherif F, Chowdhury R, Frackowiak RS, Ashburner J, Helms G, Weiskopf N, Lutti A, Draganski B (2016) New tissue priors for improved automated classification of subcortical brain structures on MRI. Neuroimage 130:157-166. 
Lövdén M, Bäckman L, Lindenberger U, Schaefer S, Schmiedek F (2010) A theoretical framework for the study of adult cognitive plasticity. Psychol Bull 136:659-676.

Lövdén M, Wenger E, Mårtensson J, Lindenberger U, Bäckman L (2013) Structural brain plasticity in adult learning and development. Neurosci Biobehav Rev 37:2296-2310.

Luque Laguna PA, Anna JE, Combes J, Streffer S, Einstein M, Timmers Steve CR, Williams F, Dell'Acqua (2020) Reproducibility, reliability and variability of fa and md in the older healthy population: a test-retest multiparametric analysis. Neuroimage Clin 26:102168.

Mandera P, Keuleers E, Wodniecka Z, Brysbaert M (2015) Subtlex-pl: subtitle-based word frequency estimates for Polish. Behav Res Methods 47:471-483.

Matuszewski J, Kossowski B, Bola Ł, Banaszkiewicz A, Paplińska M, Gyger L, Kherif F, Szwed M, Frackowiak RS, Jednoróg K, Draganski B, Marchewka A (2021) Brain plasticity dynamics during tactile braille learning in sighted subjects: multi-contrast MRI approach. Neuroimage 227:117613.

May A, Draganski B (2008) Training-induced structural changes in the adult human brain. Behav Brain Res 192:137-142.

Merabet LB, Hamilton R, Schlaug G, Swisher JD, Kiriakopoulos ET, Pitskel NB, Kauffman T, Pascual-Leone A (2008) Rapid and reversible recruitment of early visual cortex for touch. PLoS One 3:e3046.

Metzler-Baddeley C, Foley S, de Santis S, Charron C, Hampshire A, Caeyenberghs K, Jones DK (2017) Dynamics of white matter plasticity underlying working memory training: multimodal evidence from diffusion MRI and relaxometry. J Cogn Neurosci 29:1509-1520.

Morey RD (2008) Confidence intervals from normalized data: a correction to Cousineau (2005). Tutor Quant Methods Psychol 4:61-64.

Nilsson M, van Westen D, Ståhlberg F, Sundgren PC, Lätt J (2013) The role of tissue microstructure and water exchange in biophysical modeling of diffusion in white matter. MAGMA 26:345-370.

Novikov DS, Kiselev VG, Jespersen SN (2018) On modeling. Magn Reson Med 79:3172-3193.

Oishi K, Faria A, MoriVan Zijl PC, Mori S (2010) MRI atlas of human white matter, Ed 2. Amsterdam: Elsevier.

Panagiotaki E, Schneider T, Siow B, Hall MG, Lythgoe MF, Alexander DGC (2012) Compartment models of the diffusion MR signal in brain white matter: a taxonomy and comparison. Neuroimage 59:2241-2254.

Pierpaoli C, Jezzard P, Basser PJ, Barnett A, Di Chiro G (1996) Diffusion tensor MR imaging of the human brain. Radiology 201:637-648.

Pleger B, Foerster AF, Ragert P, Dinse HR, Schwenkreis P, Malin JP, Nicolas $\mathrm{V}$, Tegenthoff $\mathrm{M}$ (2003) Functional imaging of perceptual learning in human primary and secondary somatosensory cortex. Neuron 40:643653.

Qian C, Tan F (2017) Internal Capsule: The Homunculus Distribution in the Posterior Limb. Brain Behav 7:e00629.

R Development Core Team (2019) R: A Language and Environment for Statistical Computing. Vienna, Austria: R Foundation for Statistical Computing.

Sadato N, Okada T, Honda M, Yonekura Y (2002) Critical period for crossmodal plasticity in blind humans: a functional MRI study. NeuroImage 16:389-400

Sagi Y, Tavor I, Hofstetter S, Tzur-Moryosef S, Blumenfeld-Katzir T, Assaf Y (2012) Learning in the fast lane: new insights into neuroplasticity. Neuron 73:1195-1203.

Saito DN, Okada T, Honda M, Yonekura Y, Sadato N (2006) Practice makes perfect: the neural substrates of tactile discrimination by Mah-Jong experts include the primary visual cortex. BMC Neurosci 7:79.

Sampaio-Baptista C, Johansen-Berg H (2017) White matter plasticity in the adult brain. Neuron 96:1239-1251.

Sampaio-Baptista C, Khrapitchev AA, Foxley S, Schlagheck T, Scholz J, Jbabdi S, DeLuca GC, Miller KL, Taylor A, Thomas N, Kleim J, Sibson
NR, Bannerman D, Johansen-Berg H (2013) Motor skill learning induces changes in white matter microstructure and myelination. J Neurosci 33:19499-19503.

Sampaio-Baptista C, Vallès A, Khrapitchev AA, Akkermans G, Winkler AM, Foxley S, Sibson NR, Roberts M, Miller K, Diamond ME, Martens GJM, De Weerd P, Johansen-Berg H (2020) White matter structure and myelin-related gene expression alterations with experience in adult rats. Prog Neurobiol 187:101770.

Sathian K, Lacey S, Stilla R, Gibson GO, Deshpande G, Hu X, LaConte S, Glielmi C (2011) Dual pathways for haptic and visual perception of spatial and texture information. Neuroimage 57:462-475.

Schmidt-Wilcke T, Wulms N, Heba S, Pleger B, Puts NA, Glaubitz B, Kalisch T, Tegenthoff M, Dinse HR (2018) Structural changes in brain morphology induced by brief periods of repetitive sensory stimulation. Neuroimage 165:148-157.

Scholz J, Klein MC, Behrens TEJ, Johansen-Berg H (2009) Training induces changes in white-matter architecture. Nat Neurosci 12:1370-1371.

Searle SR, Speed FM, Milliken GA (1980) Population marginal means in the linear model: an alternative to least squares means. Am Stat 34:216-221.

Sexton CE, Walhovd KB, Storsve AB, Tamnes CK, Westlye LT, JohansenBerg H, Fjell AM (2014) Accelerated changes in white matter microstructure during aging: a longitudinal diffusion tensor imaging study. J Neurosci 34:15425-15436.

Siuda-Krzywicka K, Bola Ł, Paplińska M, Sumera E, Jednoróg K, Marchewka A, Śliwińska MW, Amedi A, Szwed M (2016) Massive cortical reorganization in sighted braille readers. eLife 5:e10762.

Snook L, Paulson L-A, Roy D, Phillips L, Beaulieu C (2005) Diffusion tensor imaging of neurodevelopment in children and young adults. NeuroImage 26:1164-1173.

Song SK, Yoshino J, Le TQ, Lin SJ, Sun SW, Cross AH, Armstrong RC (2005) Demyelination increases radial diffusivity in corpus callosum of mouse brain. Neuroimage 26:132-140.

Stilla R, Sathian K (2008) Selective visuo-haptic processing of shape and texture. Hum Brain Mapp 29:1123-1138.

Taubert M, Draganski B, Anwander A, Müller K, Horstmann A, Villringer A, Ragert P (2010) Dynamic properties of human brain structure: learning-related changes in cortical areas and associated fiber connections. J Neurosci 30:11670-11677.

Tuch DS, Reese TG, Wiegell MR, Makris N, Belliveau JW, Wedeen VJ (2002) High angular resolution diffusion imaging reveals intravoxel white matter fiber heterogeneity. Magn Reson Med 48:577-582.

Vos SB, Jones DK, Viergever MA, Leemans A (2011) Partial volume effect as a hidden covariate in DTI analyses. Neuroimage 55:1566-1576.

Veraart J, Sijbers J, Sunaert S, Leemans A, Jeurissen B (2013) Weighted linear least squares estimation of diffusion MRI parameters: strengths, limitations, and pitfalls. Neuroimage 81:335-346.

Veraart J, Fieremans E, Novikov DS (2016) Diffusion MRI noise mapping using random matrix theory. Magn Reson Med 76:1582-1593.

Veraart J, Raven EP, Edwards LJ, Weiskopf N, Jones DK (2021) The variability of MR axon radii estimates in the human white matter. Hum Brain Mapp 42:2201-2213.

Weber B, Koschutnig K, Schwerdtfeger A, Rominger C, Papousek I, Weiss EM, Tilp M, Fink A (2019) Learning unicycling evokes manifold changes in gray and white matter networks related to motor and cognitive functions. Sci Rep 9:4324.

Zangaladze A, Epstein CM, Grafton ST, Sathian K (1999) Involvement of visual cortex in tactile discrimination of orientation. Nature 401:587-590.

Zangenehpour S, Zatorre RJ (2010) Crossmodal recruitment of primary visual cortex following brief exposure to bimodal audiovisual stimuli. Neuropsychologia 48:591-600.

Zatorre RJ, Fields RD, Johansen-Berg H (2012) Plasticity in gray and white: neuroimaging changes in brain structure during learning. Nat Neurosci $15: 528-536$ 